\title{
Design Criterion of Polarization-Time Codes for Optical Fiber Channels
}

\author{
Elie Awwad, Ghaya Rekaya-Ben Othman and Yves Jaouën \\ TELECOM ParisTech, 46 rue Barrault, 75013 Paris, France \\ E-mail: \{awwad,rekaya,jaouen\}@ telecom-paristech.fr
}

\begin{abstract}
Coherent detection with Polarization Multiplexing (PolMux) is the most promising technique for future optical fiber transmission systems. However, the optical channel suffers from non-unitary impairments known as Polarization Dependent Loss (PDL). Space-Time coding, originally designed for wireless Rayleigh fading channels, was proven to be capable of mitigating PDL. Coding gains of ST codes were evaluated through simulations and experiments that showed differences in their performance on the optical channel and on the wireless channel. In this paper, we derive an upper bound of the pairwise error probability of an optical channel considering the PDL effect. This upper bound explains the performance of ST codes used to mitigate PDL and yields the design criterion that a code should satisfy in order to completely mitigate PDL.
\end{abstract}

Index Terms-Polarization Dependent Loss, Error Probability, Polarization-Time Coding, Optical Fiber Communication, MIMO

\section{INTRODUCTION}

From long-haul links to metropolitan and access networks, optical fibers are today's indispensable carriers of data traffic over the world. They are able to transport light over long distances with a low attenuation. Due to this property and to the $\mathrm{THz}$ bandwidth in the near infrared region of the electromagnetic spectrum, binary modulation schemes such as On-Off Keying carrying $1 \mathrm{bit} / \mathrm{symbol}$ were first used to send data on fiber-optic systems. However, the rapidly growing Internet traffic urged the search for techniques that increase the capacity of the optical fiber channel. For this purpose, coherent detection started replacing conventional direct detection schemes. The main advantages of coherent detection are the enhanced receiver sensitivity and the introduction of multilevel modulation formats that achieve high spectral efficiency. Moreover, research in Digital Signal Processing (DSP) algorithms and development of high-speed electronics offered the possibility of compensating for fiber impairments in the electrical domain at the receiver rather than optically [1]-[3], allowing the implementation of forward error correction codes and advanced equalization techniques in a DSP-based emitter and receiver.

Apart from the amplitude and the phase of an electromagnetic field, the polarization state is an available degree of freedom that is not yet used. With Polarization Multiplexing (PolMux) [4], [5], we can double the spectral efficiency of an optical fiber link by sending independent information on two orthogonal polarization states. However, during propagation through the optical channel, the transmitted signal suffers several impairments. We focus on the linear impairments that include dispersive effects: deterministic Chromatic Dispersion (CD) and random Polarization Mode Dispersion (PMD), as well as fiber attenuation and random fading effects known as Polarization Dependent Loss (PDL). To mitigate the linear impairments of the optical channel and recover the transmitted data, a polarization diversity coherent receiver along with DSP algorithms are needed [3]-[5]. While dispersive effects have been thoroughly studied and completely compensated, mitigation of PDL was still not considered and system margins were left in order to absorb the induced penalties.

A PolMux scheme can be described as a $2 \times 2$ Multi-InputMulti-Output (MIMO) system where 2 polarization states are used at the emission and the reception [6]. Instead of the spatial dimension (antennas) in a wireless scheme, we use here the polarization dimension. Hence, Space-Time (ST) coding can be applied to mitigate PDL by taking advantage of the diversity of the MIMO configuration. The motivation behind the use of ST codes, or more appropriately Polarization-Time (PT) codes in this context, is the random nature of PDL. Indeed, some optical elements attenuate unequally the polarization states of the transmitted signal. This along with random polarization rotations results in breaking the orthogonality of these states. Therefore, it is more likely to recover the original data symbols by sending linear combinations of these symbols at different time slots and on two different polarizations.

The performance of ST codes such as the Golden code [7], Silver code [8] and Alamouti code [9] was evaluated on an optical channel with PDL through simulations [10] and experimental demonstrations [11]. The Silver code performed better than the Golden code, unlike the case of the wireless Rayleigh fading channel. Besides, it was found that the performance of Alamouti code is independent of the amount of PDL in the link [12]. In this work, we aim to explain these observations through error probability calculus. We also check whether the Silver code is the optimal code for an optical channel with PDL or better coding schemes can be found.

Accordingly, the paper is organized as follows: in Section II, we recall the main linear impairments of an optical fiber link, then we present in Section III a mathematical model of an optical channel with PDL that will be used in Section IV to compute an upper bound of the error probability expression. Afterwards, in Section V, we derive the design criterion of codes that minimize the error probability of a transmission on an optical fiber channel with PDL and we investigate in 
Section VI the performance obtained with the existing codes. Finally, we conclude and set forth the perspectives of our work.

\section{OPTICAL FIBER EFFECTS}

\section{A. Fiber attenuation \& dispersive effects}

The loss coefficient of Standard Single Mode Fibers (SSMF) is equal to $0.2 \mathrm{~dB} / \mathrm{km}$. For long-haul links spanning thousands of kilometers, optical amplifiers are periodically inserted to raise the signal power to its initially injected value at the transmitter. As for chromatic dispersion (CD) and polarization mode dispersion (PMD), both time-domain and frequencydomain equalizations exist [3]. However, a special multicarrier format, Orthogonal Frequency Division Multiplexing (OFDM) has proven to be capable of eliminating all interference caused by dispersive effects using a cyclic prefix [6].

\section{B. Polarization Dependent Loss}

While the dispersion compensation was thoroughly studied, PDL effects were not being considered until recently. In fact, PDL is mainly introduced by inline components such as amplifiers, isolators, couplers and not the fiber itself. The individual PDL value of each component is kept as low as possible. However, many of these components are found in long-haul optical links leading to a large accumulated PDL. Unlike unitary dispersive effects, PDL causes Optical Signalto-Noise Ratio (OSNR) distortions. The information-carrying polarizations experience unequal loss and crosstalk [13].

\section{OPTICAL CHANNEL MODEL}

In order to proceed with an error probability calculus, we will first mathematically define the studied optical channel.

\section{A. Optical channel with PDL}

An overview of previous works on PDL, especially by A. Mecozzi, M. Shtaif [14] and N. Gisin [15], shows that PDL must be described statistically. However, we consider in our work a simple representation, commonly found in literature [16], [17], that takes into account the OSNR inequality between two polarization states as well as their crosstalk. This representation had been used in [10] to simulate the performance of PT coding in mitigating PDL and an experimental demonstration [11] validated the obtained numerical results. PDL can be described by the following transmission matrix:

$$
\begin{aligned}
& \mathbf{H}_{P D L}=\mathbf{R}_{\alpha}\left[\begin{array}{cc}
\sqrt{1-\gamma} & 0 \\
0 & \sqrt{1+\gamma}
\end{array}\right] \mathbf{R}_{\alpha}^{-1} \\
& \text { with } \mathbf{R}_{\alpha}=\left[\begin{array}{cc}
\cos (\alpha) & -\sin (\alpha) \\
\sin (\alpha) & \cos (\alpha)
\end{array}\right]
\end{aligned}
$$

The diagonal matrix gives the imbalanced attenuation values of the least and most attenuated polarization states. The rotation matrix $\mathbf{R}_{\alpha}$ describes a mismatch between the polarization states of the incident signal and the axes of the PDL component. $\gamma$ is defined through $\Gamma_{d B}=10 \log _{10} \frac{1+\gamma}{1-\gamma}$ where $\Gamma_{d B} \geq 0$ is the PDL coefficient in $\mathrm{dB}$ and consists of the ratio between the highest and the lowest gains of the link. Hence, $0 \leq \gamma \leq 1$ with $\gamma=0$ being a PDL-free case and $\gamma=1$ corresponding to a perfect polarizer. We consider in this work constant values of $\Gamma_{d B}$ and the angles of the rotation matrices are drawn from a uniform distribution over $[0: 2 \pi]$.

\section{B. PolMux OFDM channel model}

In a PolMux OFDM transmission, an OFDM signal is sent on each polarization. At the transmitter, the information symbols are assigned to different subcarriers in the frequency domain then the signal is converted to time domain by an inverse Fast Fourier Transform (iFFT). In order to absorb the interference induced by CD and PMD, a suitable cyclic prefix (CP) is added to the beginning of each OFDM symbol. At the receiver, the $\mathrm{CP}$ is removed and an FFT operation converts the signal back into the frequency domain. Despite the redundancy introduced by the CP, this prefix eliminates all Inter-SymbolInterference and allows to correct any distortion caused by a linear dispersive channel with a single-tap frequency equalizer. Each subcarrier sees a flat channel and the received symbols are given by [6]:

$$
\mathbf{Y}_{k, i}=\exp \left(j \phi_{i}\right) \exp \left(j \phi_{D}\left(\omega_{k}\right)\right) \mathbf{H}_{k} \mathbf{X}_{k, i}+\mathbf{N}_{k, i}
$$

where $\mathbf{X}_{k, i}$ is the symbol on the $k^{t h}$ subcarrier of the $i^{t h}$ OFDM symbol. $\mathbf{Y}_{k, i}$ is the received symbol. $\phi_{i}$ is the laser phase noise, $\phi_{D}\left(\omega_{k}\right)$ is the phase shift owing to chromatic dispersion common to both polarizations. $\mathbf{H}_{k}$ is the $2 \times 2$ Jones matrix of the link, resulting from a concatenation of PMD and PDL components. $\mathbf{N}_{k, i}$ represents the additive noise.

We consider next that the $\mathrm{CP}$ is larger than the delay spread induced by $\mathrm{CD}$ and PMD. Hence, it absorbs all resulting interference and the remaining effect is the non-unitary PDL that we shall regard in the error probability calculus.

\section{DERIVATION OF AN UPPER BOUND OF THE ERROR PROBABILITY}

To get an insight on the behavior of PT codes on an optical link with PDL, we consider a PolMux OFDM transmission and use $\mathbf{H}_{P D L}$ defined in (1) as the channel matrix. Therefore, each subcarrier can be seen as a slow fading and frequency non-selective channel:

$$
\mathbf{Y}_{2 \times T}=\mathbf{H}_{P D L} \mathbf{X}_{2 \times T}+\mathbf{N}_{2 \times T}
$$

$T$ is the temporal length of the code. $\mathbf{X}$ is the PT codeword matrix. $\mathbf{Y}$ is the received matrix. When $T=1, \mathbf{X}$ and $\mathbf{Y}$ are vectors and $\mathbf{X}$ contains two QAM symbols (No Coding). When $T>1$, PT coding is used $(T=2$ for the codes investigated in this paper). The noise $\mathbf{N}$ is modeled as additive white with independent and identically distributed circular complex Gaussian components $\mathcal{C N}\left(0,2 \sigma^{2}\right)$.

According to the channel model in (3), a maximum likelihood (ML) decoder estimates $\mathbf{X}^{\prime}$ with $\mathbf{X}^{\prime \prime}$ as:

$$
\mathbf{X}^{\prime \prime}=\underset{\mathbf{X}^{\prime} \in \mathcal{C}}{\operatorname{argmin}}\left\|\mathbf{Y}-\mathbf{H}_{P D L} \mathbf{X}^{\prime}\right\|^{2}
$$

where $\mathcal{C}$ is the set of all possible codewords and $\|\cdot\|$ is the Euclidian norm. We suppose that we have perfect channel 
state information, i.e. $\mathbf{H}_{P D L}$ is known. The error probability is defined as:

$$
\begin{aligned}
P_{\text {error }} & =\operatorname{Pr}\left\{\mathbf{X}^{\prime \prime} \neq \mathbf{X}^{\prime}\right\} \\
& =\sum_{\mathbf{X}^{\prime} \in \mathcal{C}} \operatorname{Pr}\left\{\mathbf{X}^{\prime}\right\} \operatorname{Pr}\left\{\mathbf{X}^{\prime \prime} \neq \mathbf{X}^{\prime} \mid \mathbf{X}^{\prime}\right\}
\end{aligned}
$$

For equiprobable codewords, the error probability can be upper-bounded by [18]:

$$
P_{\text {error }} \leq \frac{1}{\operatorname{card}(\mathcal{C})} \sum_{\substack{\mathbf{X}^{\prime}, \mathbf{X}^{\prime \prime} \in C, \mathbf{X}^{\prime \prime} \neq \mathbf{X}^{\prime}}} \operatorname{Pr}\left(\mathbf{X}^{\prime} \rightarrow \mathbf{X}^{\prime \prime}\right)
$$

where $\operatorname{card}(\mathcal{C})$ is the cardinality of $\mathcal{C}$ and $\operatorname{Pr}\left(\mathbf{X}^{\prime} \rightarrow \mathbf{X}^{\prime \prime}\right)$ is the pairwise error probability obtained by averaging the conditional pairwise error probability defined as:

$$
\begin{aligned}
\operatorname{Pr}\left(\mathbf{X}^{\prime} \rightarrow \mathbf{X}^{\prime \prime} \mid \mathbf{H}_{P D L}\right) & =\operatorname{Pr}\left\{\left\|\mathbf{Y}-\mathbf{H}_{P D L} \mathbf{X}^{\prime \prime}\right\|^{2} \leq\right. \\
& \left.\left\|\mathbf{Y}-\mathbf{H}_{P D L} \mathbf{X}^{\prime}\right\|^{2} \mid \mathbf{X}^{\prime}, \mathbf{H}_{P D L}\right\}
\end{aligned}
$$

When computing the pairwise error probability, we suppose that $\mathbf{X}^{\prime}$ and $\mathbf{X}^{\prime \prime}$ are the only possible codewords in the codeword space. Hence, we can write the pairwise error probability in terms of the Gaussian tail function $Q$ :

$$
\begin{gathered}
\operatorname{Pr}\left(\mathbf{X}^{\prime} \rightarrow \mathbf{X}^{\prime \prime} \mid \mathbf{H}_{P D L}\right)=Q\left(\frac{\left\|\mathbf{H}_{P D L}\left(\mathbf{X}^{\prime}-\mathbf{X}^{\prime \prime}\right)\right\|}{2 \sigma}\right) \\
\text { with } Q(x)=\frac{1}{\sqrt{2 \pi}} \int_{x}^{+\infty} \exp ^{-t^{2} / 2} d t
\end{gathered}
$$

Using Chernoff's bound $Q(x) \leq \frac{1}{2} \exp ^{-\frac{x^{2}}{2}} \forall x$, and averaging over $\mathbf{H}_{P D L}$, we get:

$$
\operatorname{Pr}\left(\mathbf{X}^{\prime} \rightarrow \mathbf{X}^{\prime \prime}\right) \leq \mathbb{E}_{H}\left[\exp \left(-\frac{\left\|\mathbf{H}_{\mathbf{P D L}}\left(\mathbf{X}^{\prime}-\mathbf{X}^{\prime \prime}\right)\right\|^{2}}{8 \sigma^{2}}\right)\right]
$$

where $\mathbb{E}_{H}[]$ is the averaging operation over all possible channel realizations.

Let $\mathbf{X}=\mathbf{X}^{\prime}-\mathbf{X}^{\prime \prime}$ be the difference of two codewords and $\mathbf{D}=\left[\begin{array}{cc}\sqrt{1-\gamma} & 0 \\ 0 & \sqrt{1+\gamma}\end{array}\right]$, we have:

$$
\begin{aligned}
\left\|\mathbf{H}_{P D L}\left(\mathbf{X}^{\prime}-\mathbf{X}^{\prime \prime}\right)\right\|^{2}=\left\|\mathbf{H}_{P D L} \mathbf{X}\right\|^{2} & =\left\|\mathbf{R}_{\alpha} \mathbf{D R}_{\alpha}^{-1} \mathbf{X}\right\|^{2} \\
& =\left\|\mathbf{D} \mathbf{R}_{\alpha}^{-1} \mathbf{X}\right\|^{2}
\end{aligned}
$$

Now, let $\beta=-\alpha$. We can write $\mathbf{R}_{\beta} \mathbf{X}=\mathbf{U}=\left(\begin{array}{c}\vec{v}_{1} \\ \vec{v}_{2}\end{array}\right), \vec{v}_{1,2}$ being row vectors, we get:

$$
\|\mathbf{D U}\|^{2}=\left\|\vec{v}_{1}\right\|^{2}+\left\|\vec{v}_{2}\right\|^{2}+\gamma\left(\left\|\vec{v}_{2}\right\|^{2}-\left\|\vec{v}_{1}\right\|^{2}\right)
$$

However, $\left\|\vec{v}_{1}\right\|^{2}+\left\|\vec{v}_{2}\right\|^{2}=\|\mathbf{U}\|^{2}=\|\mathbf{X}\|^{2}$ because a rotation matrix is unitary. Besides, we develop $\left\|\vec{v}_{2}\right\|^{2}-\left\|\vec{v}_{1}\right\|^{2}$ and obtain:

$$
\begin{aligned}
\left\|\vec{v}_{2}\right\|^{2}-\left\|\vec{v}_{1}\right\|^{2}=f(\beta)= & \left(\left\|\vec{x}_{2}\right\|^{2}-\left\|\vec{x}_{1}\right\|^{2}\right) \cos (2 \beta) \\
& +2 \operatorname{Re}\left(\left\langle\vec{x}_{1}, \vec{x}_{2}\right\rangle\right) \sin (2 \beta)
\end{aligned}
$$

with $\mathbf{X}=\left(\begin{array}{c}\vec{x}_{1} \\ \vec{x}_{2}\end{array}\right), \vec{x}_{1,2}$ being row vectors. Let:

$$
a=\left\|\vec{x}_{2}\right\|^{2}-\left\|\vec{x}_{1}\right\|^{2}
$$

$$
b=2 \operatorname{Re}\left(\left\langle\vec{x}_{1}, \vec{x}_{2}\right\rangle\right)
$$

Substituting (12) to (15) in (10), we get:

$$
\begin{aligned}
& \operatorname{Pr}\left(\mathbf{X}^{\prime} \rightarrow \mathbf{X}^{\prime \prime}\right)=\mathbb{E}_{H}\left[\operatorname{Pr}\left(\mathbf{X}^{\prime} \rightarrow \mathbf{X}^{\prime \prime} \mid \mathbf{H}_{P D L}\right)\right] \\
& \leq \exp \left(-\frac{\|\mathbf{X}\|^{2}}{8 \sigma^{2}}\right) \mathbb{E}_{H}\left[\exp \left(-\frac{\gamma}{8 \sigma^{2}}(a \cos (2 \beta)+b \sin (2 \beta))\right)\right]
\end{aligned}
$$

The last step consists of averaging over $\mathbf{H}_{P D L}$. Considering constant values of $\Gamma_{d B}$, we are left with one random variable $\beta$ that varies uniformly on the interval $[0: 2 \pi]$. Using the following trigonometric identity:

$$
\begin{aligned}
\operatorname{acos}(2 \beta)+b \sin (2 \beta) & =\sqrt{a^{2}+b^{2}} \cos (2 \beta+\phi) \\
\text { with } \phi & =\left\{\begin{array}{c}
-\arctan \left(\frac{b}{a}\right) \text { if } a>0 \\
\pi-\arctan \left(\frac{b}{a}\right) \text { if } a<0
\end{array}\right\}
\end{aligned}
$$

We obtain:

$$
\begin{aligned}
& \operatorname{Pr}\left(\mathbf{X}^{\prime} \rightarrow \mathbf{X}^{\prime \prime}\right) \leq \\
& \exp \left(-\frac{\|\mathbf{X}\|^{2}}{8 \sigma^{2}}\right) \frac{1}{2 \pi} \int_{\phi}^{2 \pi+\phi} \exp \left(-\frac{\gamma}{8 \sigma^{2}} \sqrt{a^{2}+b^{2}} \cos (u)\right) d u
\end{aligned}
$$

The function inside the integral is periodic of period $2 \pi$ and has an axis of symmetry $u=\pi$ in the interval $[0: 2 \pi]$. Using these two properties, (18) becomes:

$$
\begin{aligned}
& \operatorname{Pr}\left(\mathbf{X}^{\prime} \rightarrow \mathbf{X}^{\prime \prime}\right) \leq \\
& \exp \left(-\frac{\|\mathbf{X}\|^{2}}{8 \sigma^{2}}\right) \frac{1}{\pi} \int_{0}^{\pi} \exp \left(-\frac{\gamma}{8 \sigma^{2}} \sqrt{a^{2}+b^{2}} \cos (u)\right) d u
\end{aligned}
$$

Hence,

$$
\operatorname{Pr}\left(\mathbf{X}^{\prime} \rightarrow \mathbf{X}^{\prime \prime}\right) \leq \exp \left(-\frac{\|\mathbf{X}\|^{2}}{8 \sigma^{2}}\right) I_{0}\left(\frac{\gamma}{8 \sigma^{2}} \sqrt{a^{2}+b^{2}}\right)
$$

where $I_{0}(k)$ is the $0^{t h}$ order modified Bessel function of the first kind.

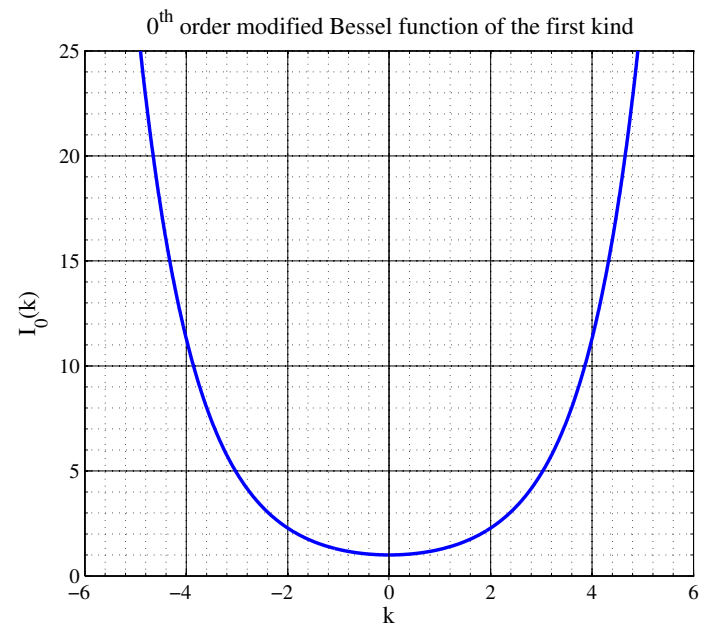

Fig. 1. Plot of the $0^{t h}$ order modified Bessel function of the first kind. 
We can approximate the error probability expression in (20) for high SNR values by using a first order approximation of $I_{0}(k)$ when $k \rightarrow \infty$ :

$$
I_{0}(k) \approx \frac{\exp (k)}{\sqrt{2 \pi k}}
$$

Then, we get:

$$
\begin{aligned}
& \exp \left(-\frac{\|\mathbf{X}\|^{2}}{8 \sigma^{2}}\right) I_{0}\left(\frac{\gamma}{8 \sigma^{2}} \sqrt{a^{2}+b^{2}}\right) \\
& \approx \exp \left(-\frac{\|\mathbf{X}\|^{2}-\gamma \sqrt{a^{2}+b^{2}}}{8 \sigma^{2}}\right)
\end{aligned}
$$

\section{DESIGN CRITERION}

In Fig. 1, we see that $I_{0}(k)$ is monotonously increasing for $k \geq 0$ and hence has its minimum for $k=0$. This corresponds to $a$ and $b$ in (20) equal to zero and as a result, the obtained error probability expression will be independent of PDL. Consequently, we define the following design criterion:

Proposition 1. A Polarization-Time code completely mitigates $P D L$ if and only if all codeword differences satisfy:

1) $a=\left\|\vec{x}_{2}\right\|^{2}-\left\|\vec{x}_{1}\right\|^{2}=0$ and

2) $b=2 \operatorname{Re}\left(\left\langle\vec{x}_{1}, \vec{x}_{2}\right\rangle\right)=0$.

When the design criterion is met, we recover the performance for two parallel additive white Gaussian channels which is the best performance we can achieve:

$$
p_{\text {error, } A W G N} \approx \exp ^{-\frac{\|\mathbf{X}\|^{2}}{8 \sigma^{2}}}
$$

We clearly see that the resulting criteria are completely different from the rank and the minimum determinant criteria for a Rayleigh fading channel [19], defining respectively a diversity gain and a coding gain. If we compare the approximation of the error probability expression at high SNR (22) to the one obtained in the case of a $2 \times 2$ MIMO Rayleigh fading channel, we notice different behaviors: the error probability of the Rayleigh fading channel decays as $\mathrm{SNR}^{-2 r}$ [19] where $r$ is the rank of the matrix $\mathbf{X}$, whereas the error probability of the PDL channel decays exponentially as a function of the SNR. Hence, Space-Time codes bring no diversity gain to the optical channel with PDL. Furthermore, if one of the design criterion is not satisfied, (22) points out that the minimum value of $\|\mathbf{X}\|^{2}-\gamma \sqrt{a^{2}+b^{2}}$ should be maximized in order to minimize the error probability.

\section{CODE PERFORMANCE ANALYSIS}

In this section, we analyze the performance of both coded and uncoded schemes using the derived upper bound of the pairwise error probability expression in (22). All the reported performance in the next subsections match with the performance presented in [10].

In Table I, we report the minimum values of $\|\mathbf{X}\|^{2}-$ $\gamma \sqrt{a^{2}+b^{2}}$ for each investigated ST code at four different PDL values. To fill the table, we set a spectral efficiency of 4 bits per channel use $(/ \mathrm{cu})$ for all coding schemes. Hence,
TABLE I

Minimum VALUe of $\|\mathbf{X}\|^{2}-\gamma \sqrt{a^{2}+b^{2}}$ FOR DIFFERENT CODING SCHEMES AT DIFFERENT PDL VALUES

\begin{tabular}{ccccc}
\hline & $\Gamma=0 d B$ & $\Gamma=3 d B$ & $\Gamma=6 d B$ & $\Gamma=10 d B$ \\
\hline Silver Code & 2 & 2 & 2 & 1.23 \\
Golden Code & 2 & 1.7 & 1.46 & 1.07 \\
No Coding & 2 & 1.34 & 0.8 & 0.38 \\
Alamouti Code & 0.8 & 0.8 & 0.8 & 0.8 \\
\hline
\end{tabular}

we use 4-QAM information symbols to build the codewords of the Silver and the Golden codes (full-rate codes with 2 symbols/cu), and 16-QAM symbols for the Alamouti code because of the redundancy in its structure $(1 \mathrm{symbol} / \mathrm{cu})$. We consider an average symbol energy $E_{S}=1$ for all constellations.

\section{A. Alamouti code}

In Fig. 2, we report the performance of Alamouti code for different PDL values $(\Gamma=3,6,10 d B)$ obtained through Monte Carlo simulations. We notice that the performance of the Alamouti code is independent of the amount of PDL in the link. The orthogonality of the Alamouti codeword matrix induces $a=b=0$ for all possible codeword differences. This explains why it performs the same independently of PDL. However, its performance is affected by the use of 16-QAM symbols giving a squared minimal distance $\|\mathbf{X}\|^{2}=0.8$.

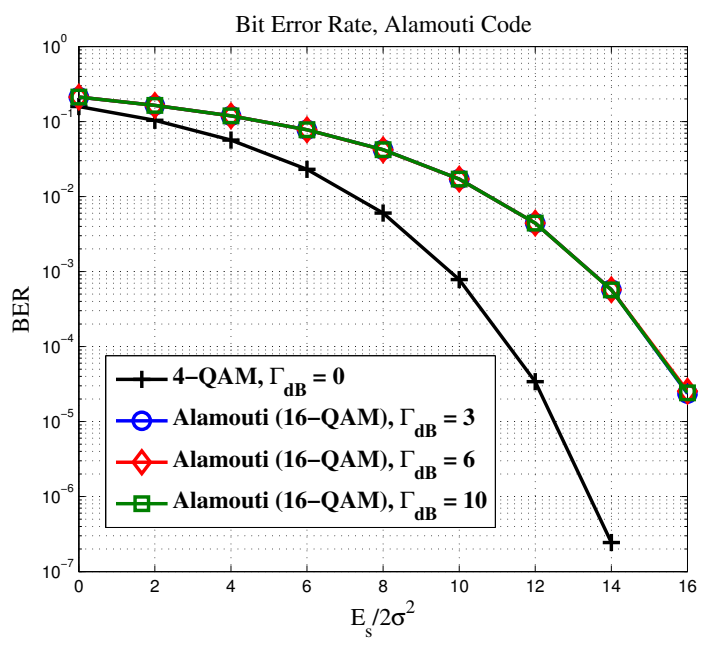

Fig. 2. Bit Error Rate as a function of SNR for the Alamouti code, obtained through Monte Carlo simulations.

\section{B. Silver code}

The Silver code is not optimal in mitigating PDL since it does not satisfy the derived design criterion. Unlike the Alamouti code, the Silver code has only some codeword differences having $a=b=0$. In Fig. 3, we plot the performance of the Silver code for different PDL values. We see that the code mitigates almost all PDL when the PDL coefficient is equal to 3 and $6 \mathrm{~dB}$. A slight difference in performance is observed for these two PDL values. Whereas for a PDL of $10 \mathrm{~dB}$, the code is not able to completely palliate PDL. Looking at Table I, we find that the minimum value of $\|\mathbf{X}\|^{2}-\gamma \sqrt{a^{2}+b^{2}}$ for the Silver code is the same at a 
PDL of 3 and $6 \mathrm{~dB}$, and is equal to 2. Whereas at a PDL of $10 \mathrm{~dB}$, this minimum falls to 1.23 which explains the obtained performance.

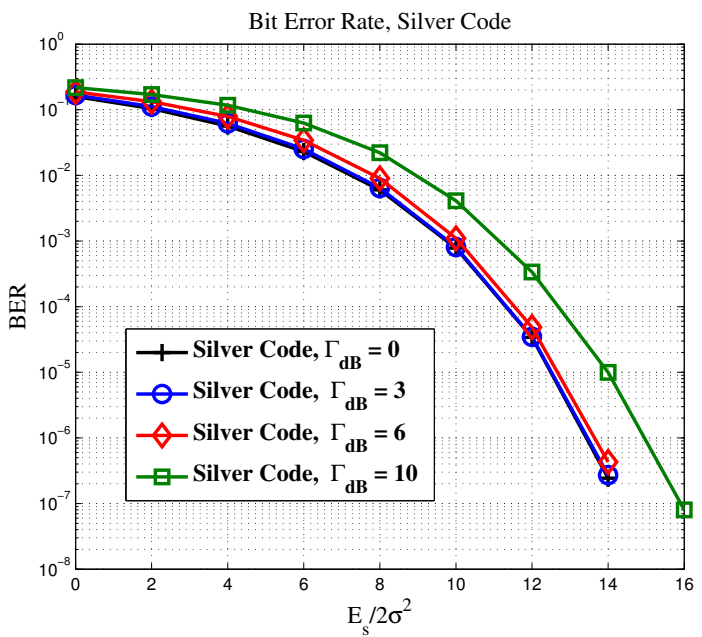

Fig. 3. Bit Error Rate as a function of SNR for the Silver code, obtained through Monte Carlo simulations.

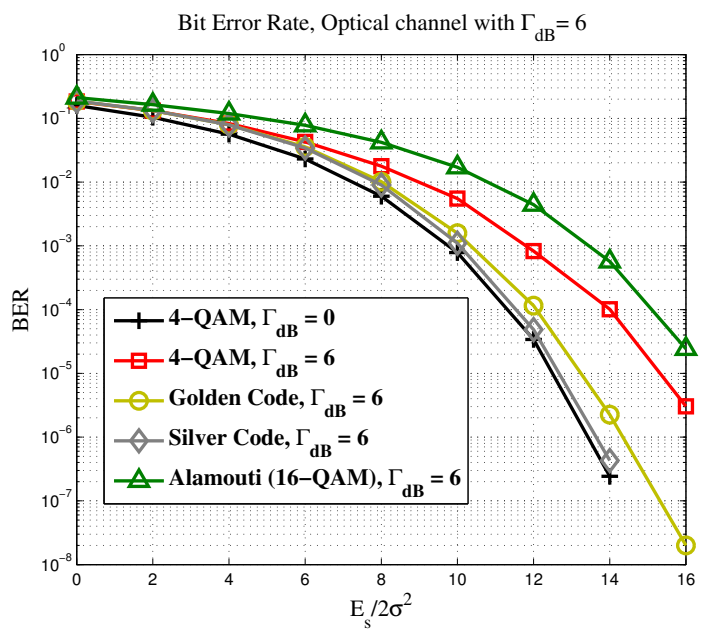

Fig. 4. Performance comparison of 4-QAM (No coding), Silver, Golden and Alamouti coding schemes when $\Gamma_{d B}=6$.

\section{Golden code and uncoded scheme}

From Fig. 4, we can see that the Silver code outperforms the Golden code for $\Gamma=6 d B$, and both reduce the penalty that PDL causes to the uncoded scheme. Again, this result can be explained by looking at Table I. Indeed, the minimum value of $\|\mathbf{X}\|^{2}-\gamma \sqrt{a^{2}+b^{2}}$ is the greatest for the Silver code followed by the Golden code and then the uncoded scheme.

In conclusion, we were able to explain, in terms of error probability bounds, the performance of the Alamouti, Silver and Golden codes when applied on an optical channel with PDL. The Golden and the Silver codes were designed to satisfy the rank and the minimum determinant criteria for a wireless channel that are no more relevant for the optical channel.

\section{Conclusion ANd Perspectives}

We have established an upper bound of the error probability expression for an optical channel with PDL that allows to predict and compare the performance of any Polarization-Time code used to mitigate PDL by computing a certain distance that depends on the codewords and the PDL value. This expression also yields the design criterion required to construct codes that guarantee a PDL-independent error probability matching the performance for an additive white Gaussian channel. In our future work, we look forward to elaborate the error probability calculus by including the statistics of the PDL coefficient $\Gamma_{d B}$ that is, under some conditions, Maxwellian distributed [14] and design specific codes for the optical channel.

\section{REFERENCES}

[1] M. G. Taylor, "Coherent detection method using DSP for demodulation of signal and subsequent equalization of propagation impairments", IEEE Photonics Technology Letters, Vol. 16, p.674-676 (2004).

[2] S. Savory, A.D. Stewart, S. Wood, G. Gavioli, M.G. Taylor, R.I. Killey, P. Bayvel, "Digital Equalization of 40Gbit/s per Wavelength Transmission over 2480km of Standard Fiber without Optical Dispersion Compensation", ECOC, pp.1-2, 2006.

[3] S. Savory,"Digital filters for coherent optical receivers", Opt. Express 16, 804-817(2008).

[4] S. Jansen, I. Morita, H. Tanaka, " $16 \times 52.5-\mathrm{Gb} / \mathrm{s}, 50-\mathrm{GHz}$ spaced, POLMUX-CO-OFDM transmission over $4,160 \mathrm{~km}$ of SSMF enabled by MIMO processing", Post-Deadline Papers, ECOC, pp.1-2, 2007.

[5] S. Jansen, I. Morita, and H. Tanaka, "10x121.9-Gb/s PDM-OFDM Transmission with 2-b/s/Hz Spectral Efficiency over 1,000 km of SSMF", in National Fiber Optic Engineers Conference, OSA, paper PDP2, 2008.

[6] W. Shieh, X. Yi, Y. Ma, and Y. Tang, "Theoretical and experimental study on PMD-supported transmission using polarization diversity in coherent optical OFDM systems", Opt. Express 15, 9936-9947 (2007).

[7] J.-C. Belfiore, G. Rekaya, E. Viterbo, "The golden code: a 2x2 fullrate space-time code with nonvanishing determinants", Information Theory, IEEE Transactions on , vol.51, no.4, pp. 1432- 1436, April 2005.

[8] O. Tirkkonen, A. Hottinen,"Improved MIMO performance with nonorthogonal space-time block codes", GLOBECOM, vol.2, pp.1122-1126, 2001.

[9] S.M. Alamouti, "A simple transmit diversity technique for wireless communications", Selected Areas in Communications, IEEE Journal on, vol.16, no.8, pp.1451-1458, Oct 1998.

[10] S. Mumtaz, G. Rekaya, Y. Jaouen, "Space-Time Codes for Optical Fiber Communication with Polarization Multiplexing”, IEEE ICC, pp.1-5, 2010.

[11] S. Mumtaz, J. Li, S. Koenig, Y. Jaouen, R. Schmogrow, G. RekayaBen Othman, and J. Leuthold, "Experimental Demonstration of PDL Mitigation using Polarization-Time Coding in PDM-OFDM Systems", in OSA, SPPCOM 2011, paper SPWB6.

[12] S. Mumtaz, G. Rekaya-Ben Othman, Y. Jaouen, J. Li, S. Koenig, R. Schmogrow, and J. Leuthold, "Alamouti Code against PDL in Polarization Multiplexed Systems", in OSA, SPPCOM 2011, paper SPTuA2.

[13] T. Duthel, C.R.S. Fludger, J. Geyer, C. Schulien, "Impact of Polarization Dependent Loss on Coherent POLMUX-NRZ-DQPSK," OFC/NFOEC 2008., pp.1-3, 24-28 Feb. 2008.

[14] A. Mecozzi, M. Shtaif, "The statistics of polarization-dependent loss in optical communication systems", Photonics Technology Letters, IEEE, vol.14, no.3, pp.313-315, March 2002.

[15] N. Gisin, "Statistics of Polarization Dependent Loss", Optics Communications 114, Elsevier (1995).

[16] F. Garcia, D. Mello, and H. Waldman, "Feedforward carrier recovery for polarization demultiplexed signals with unequal signal to noise ratios", Opt. Express 17, 7958-7969 (2009).

[17] O. Vassilieva, I. Kim, Y. Akasaka, M. Bouda, and M. Sekiya, "Interplay between PDL and nonlinear effects in coherent polarization multiplexed systems", Opt. Express 19, B357-B362 (2011).

[18] J. Proakis, M. Salehi, Digital Communications, Fifth Edition, Chapter 4, p.182-184, Mc Graw - Hill International Edition (2008).

[19] V. Tarokh, A. Naguib, N. Seshadri, A.R. Calderbank,"Space-time codes for high data rate wireless communication: performance criteria in the presence of channel estimation errors, mobility, and multiple paths", Communications, IEEE Transactions on, vol.47,no.2,pp.199-207, 1999. 